\title{
Large deviations for risk models in which each main claim induces a delayed claim
}

\author{
Claudio Macci* \\ University of Rome "Tor Vergata", ITALY
}

\begin{abstract}
In reality insurance claims may be delayed for several reasons and risk models with this feature have been discussed for some years. In this paper we present a sample path large deviation principle for the delayed claims risk model presented in [11]. Roughly speaking each main claim induces another type of claim called by-claim; any by-claim occurs later than its main claim and the time of delay is random. Successively we use Gärtner Ellis Theorem to prove a large deviation principle for a more general version of this model, in which the following items depend on the evolution of an irreducible (continuous time) Markov chain with finite state space: the intensity of the Poisson claim number process, the distribution of the claim sizes and the distribution of the random times of delay. Finally we present the Lundberg's estimate for the ruin probabilities; in the fashion of large deviations this estimate provides the exponential decay of the ruin probability as the initial capital goes to infinity.
\end{abstract}

Keywords: Large deviations; Risk model; Lundberg's estimate; Ruin probabilities; Markov modulation.

2000 Mathematics Subject Classifications: 60F10; 91B30; 62P05.

\section{Introduction}

In reality insurance claims may be delayed for several reasons. Risk models with delayed claims have been discussed for some years and a wide source of references with models having this feature can be found in [11].

In this paper we present as the "classical case" the risk model in section 2 in [11]. We also consider a generalization of this model and we use the term "Markov modulated case". Roughly speaking in both the cases we have the following situation: each main claim induces another type of claim called by-claim; any by-claim occurs later than its main claim, with a random time of delay.

We are interested in large deviation estimates for the risk models in this paper. We refer to [5] as a reference on large deviations: in particular see pages 4-5 for the definition of large deviation principle (LDP for short) and of good rate function.

Under some light tail assumptions there are some known large deviation results for a risk model with Poisson shot noise delayed claims, which coincide with the analogous results for the associated risk model with non-delayed claims: we have the same sample path large deviation rate function (see [7]) and consequently the same Lundberg's estimate for the ruin probabilities. The coincidence of Lundberg's estimates for the ruin probabilities has been already proved (see Theorem 2.1 in [3]).

For the classical case we prove a sample path LDP, namely a LDP on the space of cadlag functions $D[0,1]$. The method used in the proof is similar to the one presented in [7] for a risk

\footnotetext{
*Dipartimento di Matematica, Università di Roma "Tor Vergata", Via della Ricerca Scientifica, I-00133 Rome, Italy. e-mail: macci@mat.uniroma2.it
} 
model with Poisson shot noise delayed claims. Thus we obtain the same kind of result, i.e. we have the same rate function concerning the associated risk model with non-delayed claims (see eq. (3.1) in [11]) and consequently we have the same Lundberg's estimate for the ruin probabilities. The coincidence of Lundberg's estimates for the ruin probabilities has been already pointed out (see section 4 in [11]).

For the Markov modulated case we prove a LDP on $\mathbb{R}$ and we use the Gärtner Ellis Theorem (see section 3 of chapter 2 in [5]). The Markov modulated case is a generalization of the classical case (see Remark 3.3) and the associated risk model with non-delayed claims is a Markov modulated risk model according to the terminology in [10] (chapter 12, section 3; see also chapter 12, section 2 , subsection 2, example 4 at page 506 in the same reference).

Finally we present the Lundberg's estimate for the ruin probabilities (Proposition 4.1). More precisely we use some results in [6] to prove that, in the fashion of large deviations, the ruin probability $\psi(u)$ decays exponentially as the initial capital $u$ goes to infinity.

The results for the Markov modulated case and their proofs have some analogies with Propositions 2.1 and 3.1 in [9] which concern a risk model with Poisson shot noise delayed claims. In [9] the stochastic intensity $(\lambda(t))$ of the claim number process is more general than the Markov modulated intensity presented here, but we have less general hypotheses on the claims which are i.i.d. and independent of $(\lambda(t))$; moreover a Brownian perturbation is allowed in [9].

The paper is organized as follows. In section 2 we present the classical case and the corresponding sample path LDP. Section 3 is devoted to the Markov modulated case and, finally, we conclude with large deviation estimates for ruin probabilities in section 4.

\section{The classical case and the sample path LDP}

Let us present the model (see section 2 in [11]). Let $\left(S_{t}\right)$ be the process defined by

$$
S_{t}=u+c t-D_{t},
$$

where $u$ is the initial capital, $c$ is the (constant) premium rate and $\left(D_{t}\right)$ is the aggregate claims process. The process $\left(D_{t}\right)$ is the sum between the aggregate main claims process and the aggregate by-claims process: the first one is a compound Poisson process $\left(\sum_{k=1}^{N_{t}} X_{k}\right)$, where $\left(N_{t}\right)$ is the corresponding Poisson claims number process with intensity $\lambda$, and $N_{t}=\sum_{k \geq 1} 1_{T_{k} \leq t}$; the second one is $\left(\sum_{k \geq 1} Y_{k} 1_{T_{k}+W_{k} \leq t}\right)$, so that the corresponding claims number process $\left(\bar{N}_{t}\right)$ can be written as $\bar{N}_{t}=\sum_{k \geq 1} 1_{T_{k}+W_{k} \leq t}$. Thus

$$
D_{t}=\sum_{k=1}^{N_{t}} X_{k}+\sum_{k \geq 1} Y_{k} 1_{T_{k}+W_{k} \leq t} .
$$

Roughly speaking a main claim $X_{k}$ occurs at time $T_{k}$ and generates a by-claim $Y_{k}$ at time $T_{k}+W_{k}$, where $W_{k}$ is a random time of delay. We point out that the sequences of random variables $\left(X_{n}\right)$, $\left(Y_{n}\right),\left(T_{n}\right)$ and $\left(W_{n}\right)$ are independent; moreover each one of the sequences $\left(X_{n}\right),\left(Y_{n}\right)$ and $\left(W_{n}\right)$ is a sequence of i.i.d. positive random variables.

It is useful to consider the associated risk model with non-delayed claims; thus let us consider the modification $\left(S_{t}^{*}\right)$ of $\left(S_{t}\right)$ defined by

$$
S_{t}^{*}=u+c t-D_{t}^{*} \text {, where } D_{t}^{*}=\sum_{k=1}^{N_{t}}\left(X_{k}+Y_{k}\right) .
$$

We point out that the aggregate claims process $\left(D_{t}^{*}\right)$ is a compound Poisson process. 
In this section we consider sample path LDPs, i.e. LDPs on the space of cadlag functions $D[0,1]$. Given a process with cadlag paths $\left(Z_{t}\right)$, we say that $\left(\frac{Z_{(\alpha \cdot)}}{\alpha}\right)$ satisfies the LDP with rate function $I$ if $I: D[0,1] \rightarrow[0, \infty]$ is a lower semicontinuous function and

$$
-\inf _{f \in B^{\circ}} I(f) \leq \liminf _{\alpha \rightarrow \infty} \frac{1}{\alpha} \log P\left(\frac{Z_{(\alpha \cdot)}}{\alpha} \in B\right) \leq \limsup _{\alpha \rightarrow \infty} \frac{1}{\alpha} \log P\left(\frac{Z_{(\alpha \cdot)}}{\alpha} \in B\right) \leq-\inf _{f \in \bar{B}} I(f)
$$

for all Borel set $B$ in $D[0,1]$ (we use the standard notation $B^{\circ}$ for the interior of $B$ and $\bar{B}$ for the closure of $B$ ); thus we refer to the topology of uniform convergence. Furthermore $I$ is a good rate function if the level sets of $I$

$$
\{f \in D[0,1]: I(f) \leq \eta\}(\text { for all } \eta>0)
$$

are compact sets. by

The aim of this section is to show that $\left(\frac{D_{(\alpha \cdot)}}{\alpha}\right)$ satisfies the LDP with rate function $I$ defined

$$
I(f)=\left\{\begin{array}{ll}
\int_{0}^{1} \Lambda^{*}(\dot{f}(t)) d t & \text { if } f \in A C[0,1] \text { and } f(0)=0 \\
\infty & \text { otherwise }
\end{array},\right.
$$

where $A C[0,1]$ is the family of all absolutely continuous defined on $[0,1]$,

$$
\Lambda^{*}(x)=\sup _{\theta \in \mathbb{R}}[\theta x-\Lambda(\theta)] \text { and } \Lambda(\theta)=\lambda\left(\mathbb{E}\left[e^{\theta\left(X_{1}+Y_{1}\right)}\right]-1\right)=\lambda\left(\mathbb{E}\left[e^{\theta X_{1}}\right] \mathbb{E}\left[e^{\theta Y_{1}}\right]-1\right) .
$$

This LDP will be proved assuming that the next light tail condition (superexponential condition) holds:

(S1): $\mathbb{E}\left[e^{\theta X_{1}}\right] \mathbb{E}\left[e^{\theta Y_{1}}\right]<\infty$ for all $\theta \in \mathbb{R}$.

The idea is to prove the sample path LDP by adapting the procedure in [7] for the Poisson shot noise delayed claims process. Thus we shall show that $\left(\frac{D_{(\alpha \cdot)}^{*}}{\alpha}\right)$ and $\left(\frac{D_{(\alpha \cdot)}}{\alpha}\right)$ are exponentially equivalent as $\alpha \rightarrow \infty$ (see Definition 4.2.10 in [5]), so that the sample path LDP we want to prove will follow from Theorem 4.2.13 in [5] and the next known result (see [2]; see also [4] and the references cited therein).

Proposition 2.1 Assume (S1) holds. Then $\left(\frac{D_{(\alpha \cdot)}^{*}}{\alpha}\right)$ satisfies the LDP with rate function I as in (4). Moreover the rate function I is good.

We shall use the symbol [.] to denote the integer part of a real number. Let $\left(A_{n}\right)$ be the sequence defined by

$$
A_{n}=\sum_{k=1}^{n} Y_{k} 1_{W_{k}>T_{k}} .
$$

In view of the proof of the sample path LDP the following lemma is needed.

Lemma 2.2 Assume (S1) holds. Then $\lim _{n \rightarrow \infty} \frac{1}{n} \log \mathbb{E}\left[e^{\theta A_{n}}\right]=0$ for all $\theta>0$.

Proof. Let $\theta>0$ be arbitrarily fixed. We have $\mathbb{E}\left[e^{\theta A_{n}}\right] \geq 1$ for all $n \geq 1$ since $\theta>0$ and the random variable $A_{n}$ is nonnegative; thus we can immediately say that $\liminf _{n \rightarrow \infty} \frac{1}{n} \log \mathbb{E}\left[e^{\theta A_{n}}\right] \geq 0$. Thus we complete the proof showing that $\limsup _{n \rightarrow \infty} \frac{1}{n} \log \mathbb{E}\left[e^{\theta A_{n}}\right] \leq 0$.

By the dominated convergence theorem we have $\lim _{t \rightarrow \infty} \mathbb{E}\left[e^{\theta Y_{1} 1_{W_{1}>t}}\right]=1$; therefore for all $\varepsilon>0$ there exists $t(\varepsilon, \theta)>0$ such that

$$
\mathbb{E}\left[e^{\theta Y_{1} 1_{W_{1}>t}}\right]<e^{\varepsilon} \text { for all } t \geq t(\varepsilon, \theta) .
$$


For all $n \geq 1$ and for all $\eta>0$ we have

$$
P\left(N_{t(\varepsilon, \theta)} \geq[n \varepsilon]\right) \leq e^{-\eta[n \varepsilon]} \mathbb{E}\left[e^{\eta N_{t(\varepsilon, \theta)}}\right]=e^{-\eta[n \varepsilon]+\lambda t(\varepsilon, \theta)\left(e^{\eta}-1\right)},
$$

and therefore $\lim \sup _{n \rightarrow \infty} \frac{1}{n} \log P\left(N_{t(\varepsilon, \theta)} \geq[n \varepsilon]\right) \leq-\eta \varepsilon$; thus, since $\eta>0$ is arbitrary,

$$
\lim _{n \rightarrow \infty} \frac{1}{n} \log P\left(N_{t(\varepsilon, \theta)} \geq[n \varepsilon]\right)=-\infty .
$$

In conclusion, since we have $P\left(T_{[n \varepsilon]} \leq t(\varepsilon, \theta)\right)=P\left(N_{t(\varepsilon, \theta)} \geq[n \varepsilon]\right)$ for all $n \geq 1$, we obtain

$$
\lim _{n \rightarrow \infty} \frac{1}{n} \log P\left(T_{[n \varepsilon]} \leq t(\varepsilon, \theta)\right)=-\infty .
$$

Now let us write

$$
\mathbb{E}\left[e^{\theta A_{n}}\right]=\mathbb{E}\left[e^{\theta A_{n}} 1_{T_{[n \varepsilon]}<t(\varepsilon, \theta)}\right]+\mathbb{E}\left[e^{\theta A_{n}} 1_{T_{[n \varepsilon]} \geq t(\varepsilon, \theta)}\right]
$$

and we shall derive bounds on each addendum. Note that $A_{n} \leq \sum_{k=1}^{n} Y_{k}$ and we have

$$
\mathbb{E}\left[e^{\theta A_{n}} 1_{T_{[n \varepsilon]}<t(\varepsilon, \theta)}\right] \leq \mathbb{E}\left[e^{\theta \sum_{k=1}^{n} Y_{k}} 1_{T_{[n \varepsilon]}<t(\varepsilon, \theta)}\right]=\left(\mathbb{E}\left[e^{\theta Y_{1}}\right]\right)^{n} P\left(T_{[n \varepsilon]}<t(\varepsilon, \theta)\right)
$$

since $\theta>0,\left(Y_{1}, \ldots, Y_{n}, T_{[n \varepsilon]}\right)$ are independent and $\left(Y_{1}, \ldots, Y_{n}\right)$ are i.i.d.; thus we obtain

$$
\lim _{n \rightarrow \infty} \frac{1}{n} \log \mathbb{E}\left[e^{\theta A_{n}} 1_{T_{[n \varepsilon]}<t(\varepsilon, \theta)}\right]=-\infty
$$

holds by (8) and (S1). Moreover set

$$
B_{n}=\sum_{k=1}^{[n \varepsilon]-1} Y_{k}+\sum_{k=[n \varepsilon]}^{n} Y_{k} 1_{W_{k}>t(\varepsilon, \theta)}
$$

and, if $\left\{T_{[n \varepsilon]} \geq t(\varepsilon, \theta)\right\}$, we have $A_{n} \leq B_{n}$; thus

$$
\mathbb{E}\left[e^{\theta A_{n}} 1_{T_{[n \varepsilon]} \geq t(\varepsilon, \theta)}\right] \leq \mathbb{E}\left[e^{\theta B_{n}} 1_{T_{[n \varepsilon]} \geq t(\varepsilon, \theta)}\right] \leq\left(\mathbb{E}\left[e^{\theta Y_{1}}\right]\right)^{[n \varepsilon]-1} e^{\varepsilon(n-[n \varepsilon]+1)}
$$

where the first inequality holds by $A_{n} \leq B_{n}$ (under the hypothesis $\left\{T_{[n \varepsilon]} \geq t(\varepsilon, \theta)\right\}$ ) and the second inequality holds by the definition of $B_{n}$ and (7).

Thus, by (9) and (10), we have $\limsup _{n \rightarrow \infty} \frac{1}{n} \log \mathbb{E}\left[e^{\theta A_{n}}\right] \leq \varepsilon \log \mathbb{E}\left[e^{\theta Y_{1}}\right]+\varepsilon(1-\varepsilon)$. In conclusion, since (S1) holds and $\varepsilon>0$ can be chosen arbitrarily small, we have $\lim _{\sup } \rightarrow \infty \frac{1}{n} \log \mathbb{E}\left[e^{\theta A_{n}}\right] \leq 0$.

Now we are able to complete the proof of the sample path LDP.

Proposition 2.3 Assume (S1) holds. Then $\left(\frac{D_{(\alpha \cdot)}}{\alpha}\right)$ satisfies the LDP with rate function I as in (4).

Proof. Proposition 2.1 provides the LDP of $\left(\frac{D_{(\alpha \cdot)}^{*}}{\alpha}\right)$ and the goodness of the rate function $I$ in (4). Thus, by Theorem 4.2.13 in [5], we only need to show that $\left(\frac{D_{(\alpha \cdot)}}{\alpha}\right)$ and $\left(\frac{D_{(\alpha \cdot)}^{*}}{\alpha}\right)$ are exponentially equivalent as $\alpha \rightarrow \infty$, i.e.

$$
\lim _{\alpha \rightarrow \infty} \frac{1}{\alpha} \log P\left(M_{\alpha}>\delta\right)=-\infty(\text { for all } \delta>0) \text {, where } M_{\alpha}=\frac{1}{\alpha} \sup _{t \in[0,1]}\left|D_{\alpha t}^{*}-D_{\alpha t}\right| .
$$


Let $\delta>0$ be arbitrarily fixed. We point out that $D_{t}^{*} \geq D_{t}$ for all $t \geq 0$ and the supremum $M_{\alpha}$ is attained at some epoch $T_{n} \in[0, \alpha]$ concerning the underlying Poisson process $\left(N_{t}\right)$; thus

$$
M_{\alpha}=\frac{1}{\alpha} \max _{n: T_{n} \in[0, \alpha]} \sum_{k=1}^{n} Y_{k}-\sum_{k=1}^{n} Y_{k} 1_{T_{k}+W_{k} \leq T_{n}}
$$

whence

$$
M_{\alpha}=\frac{1}{\alpha} \max _{n: T_{n} \in[0, \alpha]} \sum_{k=1}^{n} Y_{k} 1_{T_{k}+W_{k}>T_{n}} .
$$

Since $T_{n}$ is the sum of $n$ exponential random variables with mean $\frac{1}{\lambda}$, for all $\eta>0$ and for all integers $K>\lambda$ we have

$$
P\left(T_{K[\alpha]}<\alpha\right) \leq e^{\eta \alpha} \mathbb{E}\left[e^{\left.-\eta T_{K[\alpha]}\right]}=e^{\eta \alpha}\left(\frac{\lambda}{\lambda+\eta}\right)^{K[\alpha]}\right.
$$

and therefore

$$
\lim _{\alpha \rightarrow \infty} \frac{1}{\alpha} \log P\left(T_{K[\alpha]}<\alpha\right) \leq \eta+K \log \frac{\lambda}{\lambda+\eta}(\text { for all } \eta>0) .
$$

Furthermore we have the following inequalities:

$$
\begin{gathered}
P\left(M_{\alpha}>\delta, T_{K[\alpha]} \geq \alpha\right) \leq P\left(\max _{1 \leq n \leq K[\alpha]} \sum_{k=1}^{n} Y_{k} 1_{T_{k}+W_{k}>T_{n}}>\alpha \delta\right) \leq \\
\leq K[\alpha] \max _{1 \leq n \leq K[\alpha]} P\left(\sum_{k=1}^{n} Y_{k} 1_{T_{k}+W_{k}>T_{n}}>\alpha \delta\right) ;
\end{gathered}
$$

they follow from (12) and the union bound respectively.

Now let us remark that (=law means "equally distributed")

$$
\begin{gathered}
\sum_{k=1}^{n} Y_{k} 1_{T_{k}+W_{k}>T_{n}}=\sum_{k=1}^{n} Y_{k} 1_{W_{k}>T_{n}-T_{k}}={ }^{l a w} \sum_{k=1}^{n} Y_{k} 1_{W_{k}>T_{n-k}}= \\
=\sum_{k=1}^{n} Y_{n-k} 1_{W_{n-k}>T_{k}}={ }^{l a w} \sum_{k=1}^{n} Y_{k} 1_{W_{k}>T_{k}}={ }^{\text {by }(6)} A_{n} ;
\end{gathered}
$$

thus we can rewrite the above inequality as

$$
P\left(M_{\alpha}>\delta, T_{K[\alpha]} \geq \alpha\right) \leq K[\alpha] \max _{1 \leq n \leq K[\alpha]} P\left(A_{n}>\alpha \delta\right),
$$

and we obtain

$$
P\left(M_{\alpha}>\delta, T_{K[\alpha]} \geq \alpha\right) \leq K[\alpha] P\left(A_{K[\alpha]}>\alpha \delta\right)
$$

since $\left(A_{n}\right)$ is an increasing sequence. Thus, for all $\theta>0$,

$$
P\left(M_{\alpha}>\delta, T_{K[\alpha]} \geq \alpha\right) \leq K[\alpha] P\left(A_{K[\alpha]}>\alpha \delta\right) \leq K[\alpha] e^{-\theta \alpha \delta} \mathbb{E}\left[e^{\theta A_{K[\alpha]}}\right]
$$

and, by Lemma $2.2, \lim \sup _{\alpha \rightarrow \infty} \frac{1}{\alpha} \log P\left(M_{\alpha}>\delta, T_{K[\alpha]} \geq \alpha\right) \leq-\theta \delta$. In conclusion

$$
\lim _{\alpha \rightarrow \infty} \frac{1}{\alpha} \log P\left(M_{\alpha}>\delta, T_{K[\alpha]} \geq \alpha\right)=-\infty
$$

holds since $\theta>0$ is arbitrarily chosen.

Now, by the union bound, we get

$$
P\left(M_{\alpha}>\delta\right) \leq P\left(M_{\alpha}>\delta, T_{K[\alpha]} \geq \alpha\right)+P\left(T_{K[\alpha]}<\alpha\right)
$$


hence, by (13) and (14), we have

$$
\limsup _{\alpha \rightarrow \infty} \frac{1}{\alpha} \log P\left(M_{\alpha}>\delta\right) \leq \eta+K \log \frac{\lambda}{\lambda+\eta}(\text { for all } \eta>0) .
$$

Then

$$
\limsup _{\alpha \rightarrow \infty} \frac{1}{\alpha} \log P\left(M_{\alpha}>\delta\right) \leq \inf _{\eta>0}\left\{\eta+K \log \frac{\lambda}{\lambda+\eta}\right\}=K-\lambda-K \log \frac{K}{\lambda}
$$

and we obtain (11) by taking $K \rightarrow \infty$ in the latter right hand side.

\section{The Markov modulated case}

In this section we still consider the process $\left(S_{t}\right)$ as in (1) and the process $\left(S_{t}^{*}\right)$ as in (3); on the contrary we consider different (and more general) hypotheses on the process $\left(D_{t}\right)$ in $(2)$ and on the process $\left(D_{t}^{*}\right)$ in $(3)$.

Let $J=\left(J_{t}\right)$ be an irreducible continuous time Markov chain with finite state space $E$ and let $\left(p_{i j}\right)_{i, j \in E}$ be the intensity matrix of $J$. Moreover let $\left[\lambda_{i}\right]_{i \in E}$ be a vector of positive number and let $\left(G_{i}\right)_{i \in E}$ be a family of product measures on $(0, \infty) \times(0, \infty) \times(0, \infty)$; more precisely we use the notation

$$
G_{i}=G_{i}^{X} \otimes G_{i}^{Y} \otimes G_{i}^{W}
$$

where $G_{i}^{X}, G_{i}^{Y}, G_{i}^{W}$ are the marginal distributions of $G_{i}$. Then let us consider the following situation: $\left(\left(X_{n}, Y_{n}, W_{n}\right)\right)$ and $\left(N_{t}\right)$ are conditionally independent given $J ;\left(N_{t}\right)$ is a Markov modulated Poisson process, i.e. a doubly stochastic Poisson process with intensity $\left(\lambda_{J_{t}}\right) ;\left(\left(X_{n}, Y_{n}, W_{n}\right)\right)$ are independent given $J$ and, for all $n \geq 1$, the conditional distribution of $\left(X_{n}, Y_{n}, W_{n}\right)$ given $J$ is $G_{J_{T_{n}}}$.

In general we shall use the notation $\mathbb{E}_{i}[f(X, Y, W)]$ to denote the expected value of a random variable $f(X, Y, W)$, where $(X, Y, W)$ is a random variable with distribution $G_{i}$.

In view of what follows it is useful to consider the following function $L: \mathbb{R}^{E} \rightarrow \mathbb{R}$ (for details see section 2 in [1]). Let $\underline{v}=\left[v_{i}\right]_{i \in E}$ be arbitrarily fixed and let us consider the matrix $P(\underline{v})=$ $\left(p_{i j}+\delta_{i j} v_{i}\right)_{i, j \in E}$, where

$$
p_{i j}+\delta_{i j} v_{i}=\left\{\begin{array}{ll}
p_{i j}+v_{i} & \text { if } i=j \\
p_{i j} & \text { if } i \neq j
\end{array} .\right.
$$

Then Perron Frobenius Theorem guarantees the existence of a simple and positive eigenvalue denoted by $e^{L(\underline{v})}$ of the exponential matrix $e^{P(\underline{v})}$, which is equal to the spectral radius of $e^{P(\underline{v})}$. It is important to point out that

$$
L(\underline{v})=\lim _{t \rightarrow \infty} \frac{1}{t} \log \mathbb{E}\left[e^{\int_{0}^{t} v_{J_{s}} d s}\right] \text { (for all } \underline{v} \in \mathbb{R}^{E} \text { ) }
$$

whatever is the initial distribution of $J$. The function $L(\underline{v})$ is convex, nondecreasing with respect to each component $v_{i}$ of $\underline{v}$ and $\nabla L(\underline{0})=\pi$, where $\underline{0}$ is the null vector in $\mathbb{R}^{E}$ and $\pi=\left(\pi_{i}\right)_{i \in E}$ is the stationary distribution of $J$.

The aim of this section is to show that $\left(\frac{D_{t}}{t}\right)$ satisfies the LDP with rate function $\Lambda^{*}$ defined by

$$
\Lambda^{*}(x)=\sup _{\theta \in \mathbb{R}}[\theta x-\Lambda(\theta)] \text { and } \Lambda(\theta)=L\left(\left[\lambda_{i}\left(\mathbb{E}_{i}\left[e^{\theta(X+Y)}\right]-1\right)\right]_{i \in E}\right)=L\left(\left[\lambda_{i}\left(\mathbb{E}_{i}\left[e^{\theta X}\right] \mathbb{E}_{i}\left[e^{\theta Y}\right]-1\right)\right]_{i \in E}\right) .
$$

This LDP will be proved by the application of Gärtner Ellis Theorem and we assume that the next light tail condition (S2) holds (it is a generalization of (S1) presented for the classical case).

(S2): for all $i \in E$ we have $\mathbb{E}_{i}\left[e^{\theta(X+Y)}\right]=\mathbb{E}_{i}\left[e^{\theta X}\right] \mathbb{E}_{i}\left[e^{\theta Y}\right]<\infty$ for all $\theta \in \mathbb{R}$.

We can say that $\left(\frac{D_{t}^{*}}{t}\right)$ satisfies the LDP with the same rate function. Thus we have an analogy with the sample path LDP proved in the previous section. 
Proposition 3.1 Assume (S2) holds. Then $\left(\frac{D_{t}}{t}\right)$ satisfies the LDP with rate function $\Lambda^{*}$ as in (16), i.e.

$$
-\inf _{x \in B^{\circ}} \Lambda^{*}(x) \leq \liminf _{t \rightarrow \infty} \frac{1}{t} \log P\left(\frac{D_{t}}{t} \in B\right) \leq \limsup _{t \rightarrow \infty} \frac{1}{t} \log P\left(\frac{D_{t}}{t} \in B\right) \leq-\inf _{x \in \bar{B}} \Lambda^{*}(x)
$$

for all Borel set $B$ in $\mathbb{R}$ (as usual we use the standard notation $B^{\circ}$ for the interior of $B$ and $\bar{B}$ for the closure of $B$ ).

Before proving Proposition 3.1 the following Lemma is needed.

Lemma 3.2 Let us consider a random variable $Z=\sum_{n \geq 1} \varphi\left(T_{n},\left(X_{n}, Y_{n}, W_{n}\right)\right)$ according to the presentation above. Then $\mathbb{E}\left[e^{\theta Z}\right]=\mathbb{E}\left[\exp \left(\int_{0}^{\infty} \lambda_{J_{s}}\left(\mathbb{E}_{J_{s}}\left[e^{\theta \varphi(s,(X, Y, W)}\right]-1\right) d s\right)\right]$.

Proof of Lemma 3.2. This formula can be proved following the lines of the proof of Lemma 2.3 in [9]; more precisely we have to consider the extension considered in Lemma A.1 in [8].

Proof of Proposition 3.1. We want to apply Gärtner Ellis Theorem and, for all $\theta \in \mathbb{R}$, we need to check the limit

$$
\lim _{t \rightarrow \infty} \frac{1}{t} \log \mathbb{E}\left[e^{t \theta \frac{D_{t}}{t}}\right]=\Lambda(\theta),
$$

with $\Lambda(\theta)$ as in (16).

One can immediately say that (17) holds when $\theta=0$ since $\Lambda(0)=L(\underline{0})=0$. For $\theta \neq 0$ we consider Lemma 3.2 with

$$
\varphi(s,(x, y, w))=x 1_{(0, t]}(s)+y 1_{(0, t]}(s+w)
$$

for each fixed $t \geq 0$; indeed in such a case $D_{t}=\sum_{n \geq 1} \varphi\left(T_{n},\left(X_{n}, Y_{n}, W_{n}\right)\right)$ and we have

$$
\begin{aligned}
\mathbb{E}\left[e^{\theta D_{t}}\right] & =\mathbb{E}\left[\exp \left(\int_{0}^{\infty} \lambda_{J_{s}}\left(\mathbb{E}_{J_{s}}\left[e^{\theta\left[X 1_{(0, t]}(s)+Y 1_{(0, t]}(s+W)\right]}\right]-1\right) d s\right)\right]= \\
& =\mathbb{E}\left[\exp \left(\int_{0}^{t} \lambda_{J_{s}}\left(\mathbb{E}_{J_{s}}\left[e^{\theta\left[X+Y 1_{(0, t-s]}(W)\right]}\right]-1\right) d s\right)\right],
\end{aligned}
$$

whence we obtain

$$
\mathbb{E}\left[e^{\theta D_{t}}\right]=\mathbb{E}\left[\exp \left(\int_{0}^{t} \lambda_{J_{t-u}}\left(\mathbb{E}_{J_{t-u}}\left[e^{\theta\left[X+Y 1_{(0, u]}(W)\right]}\right]-1\right) d u\right)\right]
$$

by the change of variable $u=t-s$. Now let us consider the cases $\theta>0$ and $\theta<0$ separately.

Case $\theta>0$. By (18) we have

$$
\mathbb{E}\left[e^{\theta D_{t}}\right] \leq \mathbb{E}\left[\exp \left(\int_{0}^{t} \lambda_{J_{t-u}}\left(\mathbb{E}_{J_{t-u}}\left[e^{\theta(X+Y)}\right]-1\right) d u\right)\right]=\mathbb{E}\left[\exp \left(\int_{0}^{t} \lambda_{J_{s}}\left(\mathbb{E}_{J_{s}}\left[e^{\theta(X+Y)}\right]-1\right) d s\right)\right]
$$

thus

$$
\limsup _{t \rightarrow \infty} \frac{1}{t} \log \mathbb{E}\left[e^{\theta D_{t}}\right] \leq \limsup _{t \rightarrow \infty} \frac{1}{t} \log \mathbb{E}\left[\exp \left(\int_{0}^{t} \lambda_{J_{s}}\left(\mathbb{E}_{J_{s}}\left[e^{\theta(X+Y)}\right]-1\right) d s\right)\right]=\Lambda(\theta)
$$

by (15) and (16). Furthermore, by the dominated convergence theorem, for all $\varepsilon>0$ there exists $t(\varepsilon, \theta)>0$ such that

$$
\mathbb{E}_{i}\left[e^{\theta Y 1_{(0, t]}(W)}\right]>\mathbb{E}_{i}\left[e^{\theta Y}\right]-\varepsilon(\text { for all } i \in E \text { and for all } t>t(\varepsilon, \theta))
$$


whence we obtain (starting from (18))

$$
\begin{gathered}
\mathbb{E}\left[e^{\theta D_{t}}\right] \geq \mathbb{E}\left[\exp \left(\int_{t(\varepsilon, \theta)}^{t} \lambda_{J_{t-u}}\left(\mathbb{E}_{J_{t-u}}\left[e^{\theta\left[X+Y 1_{(0, u]}(W)\right]}\right]-1\right) d u\right)\right]= \\
=\mathbb{E}\left[\exp \left(\int_{t(\varepsilon, \theta)}^{t} \lambda_{J_{t-u}}\left(\mathbb{E}_{J_{t-u}}\left[e^{\theta X}\right] \mathbb{E}_{J_{t-u}}\left[e^{\theta Y 1_{(0, u]}(W)}\right]-1\right) d u\right)\right] \geq \\
\geq \mathbb{E}\left[\exp \left(\int_{t(\varepsilon, \theta)}^{t} \lambda_{J_{t-u}}\left(\mathbb{E}_{J_{t-u}}\left[e^{\theta X}\right]\left(\mathbb{E}_{J_{t-u}}\left[e^{\theta Y}\right]-\varepsilon\right)-1\right) d u\right)\right]={ }^{s=t-u} \\
=\mathbb{E}\left[\exp \left(\int_{0}^{t-t(\varepsilon, \theta)} \lambda_{J_{s}}\left(\mathbb{E}_{J_{s}}\left[e^{\theta X}\right]\left(\mathbb{E}_{J_{s}}\left[e^{\theta Y}\right]-\varepsilon\right)-1\right) d s\right)\right](\text { for all } t>t(\varepsilon, \theta))
\end{gathered}
$$

thus

$$
\liminf _{t \rightarrow \infty} \frac{1}{t} \log \mathbb{E}\left[e^{\theta D_{t}}\right] \geq L\left(\left[\lambda_{i}\left(\mathbb{E}_{i}\left[e^{\theta X}\right]\left(\mathbb{E}_{i}\left[e^{\theta Y}\right]-\varepsilon\right)-1\right)\right]_{i \in E}\right)
$$

by $(15)$ and

$$
\liminf _{t \rightarrow \infty} \frac{1}{t} \log \mathbb{E}\left[e^{\theta D_{t}}\right] \geq L\left(\left[\lambda_{i}\left(\mathbb{E}_{i}\left[e^{\theta X}\right] \mathbb{E}_{i}\left[e^{\theta Y}\right]-1\right)\right]_{i \in E}\right)=\Lambda(\theta)
$$

holds by the continuity of $L$ and by (16).

Case $\theta<0$. By $(18)$ we have

$$
\mathbb{E}\left[e^{\theta D_{t}}\right] \geq \mathbb{E}\left[\exp \left(\int_{0}^{t} \lambda_{J_{t-u}}\left(\mathbb{E}_{J_{t-u}}\left[e^{\theta(X+Y)}\right]-1\right) d u\right)\right]=\mathbb{E}\left[\exp \left(\int_{0}^{t} \lambda_{J_{s}}\left(\mathbb{E}_{J_{s}}\left[e^{\theta(X+Y)}\right]-1\right) d s\right)\right]
$$

thus

$$
\liminf _{t \rightarrow \infty} \frac{1}{t} \log \mathbb{E}\left[e^{\theta D_{t}}\right] \geq \liminf _{t \rightarrow \infty} \frac{1}{t} \log \mathbb{E}\left[\exp \left(\int_{0}^{t} \lambda_{J_{s}}\left(\mathbb{E}_{J_{s}}\left[e^{\theta(X+Y)}\right]-1\right) d s\right)\right]=\Lambda(\theta)
$$

by (15) and (16). Furthermore, by the dominated convergence theorem, for all $\varepsilon>0$ there exists $t(\varepsilon, \theta)>0$ such that

$$
\mathbb{E}_{i}\left[e^{\theta Y 1_{(0, t]}(W)}\right]<\mathbb{E}_{i}\left[e^{\theta Y}\right]+\varepsilon \text { (for all } i \in E \text { and for all } t>t(\varepsilon, \theta) \text { ) }
$$

whence we obtain (starting from (18))

$$
\begin{gathered}
\mathbb{E}\left[e^{\theta D_{t}}\right] \leq \mathbb{E}\left[\exp \left(\int_{t(\varepsilon, \theta)}^{t} \lambda_{J_{t-u}}\left(\mathbb{E}_{J_{t-u}}\left[e^{\theta\left[X+Y 1_{(0, u]}(W)\right]}\right]-1\right) d u\right)\right]= \\
=\mathbb{E}\left[\exp \left(\int_{t(\varepsilon, \theta)}^{t} \lambda_{J_{t-u}}\left(\mathbb{E}_{J_{t-u}}\left[e^{\theta X}\right] \mathbb{E}_{J_{t-u}}\left[e^{\theta Y 1_{(0, u]}(W)}\right]-1\right) d u\right)\right] \leq \\
\leq \mathbb{E}\left[\exp \left(\int_{t(\varepsilon, \theta)}^{t} \lambda_{J_{t-u}}\left(\mathbb{E}_{J_{t-u}}\left[e^{\theta X}\right]\left(\mathbb{E}_{J_{t-u}}\left[e^{\theta Y}\right]+\varepsilon\right)-1\right) d u\right)\right]=^{s=t-u} \\
=\mathbb{E}\left[\exp \left(\int_{0}^{t-t(\varepsilon, \theta)} \lambda_{J_{s}}\left(\mathbb{E}_{J_{s}}\left[e^{\theta X}\right]\left(\mathbb{E}_{J_{s}}\left[e^{\theta Y}\right]+\varepsilon\right)-1\right) d s\right)\right](\text { for all } t>t(\varepsilon, \theta))
\end{gathered}
$$

thus

$$
\limsup _{t \rightarrow \infty} \frac{1}{t} \log \mathbb{E}\left[e^{\theta D_{t}}\right] \leq L\left(\left[\lambda_{i}\left(\mathbb{E}_{i}\left[e^{\theta X}\right]\left(\mathbb{E}_{i}\left[e^{\theta Y}\right]+\varepsilon\right)-1\right)\right]_{i \in E}\right)
$$

by (15) and

$$
\limsup _{t \rightarrow \infty} \frac{1}{t} \log \mathbb{E}\left[e^{\theta D_{t}}\right] \leq L\left(\left[\lambda_{i}\left(\mathbb{E}_{i}\left[e^{\theta X}\right] \mathbb{E}_{i}\left[e^{\theta Y}\right]-1\right)\right]_{i \in E}\right)=\Lambda(\theta)
$$

holds by the continuity of $L$ and by (16). 
Remark 3.3 The Markov modulated case is a generalization of the classical case. This can be trivially explained by considering the set $E$ reduced to a single point. A more interesting way consists to consider the following condition $(C)$ and some consequences:

$(C)$ : the distributions $\left(G_{i}\right)_{i \in E}$ are all the same $G$ and the values $\left(\lambda_{i}\right)_{i \in E}$ are all the same $\lambda$.

A first consequence is that $\left(D_{t}\right)$ satisfies the same hypotheses presented for the classical case, $G$ is the common distribution the random variables $\left(\left(X_{n}, Y_{n}, W_{n}\right)\right)$, and $\left(D_{t}\right)$ and $\left(J_{t}\right)$ are independent. Furthermore (S2) coincides with (S1). Finally $\Lambda$ in (16) coincides with $\Lambda$ in (5); indeed, if for some $v \in \mathbb{R}$ we have $v_{i}=v$ for all $i \in E$, then $L\left(\left[v_{i}\right]_{i \in E}\right)=v$.

\section{Large deviation estimates for ruin probabilities}

The aim of this section is to present some large deviation estimates for the ruin probabilities $(\psi(u))_{u>0}$ defined by

$$
\psi(u)=P\left(\tau_{u}<\infty\right), \text { where } \tau_{u}=\inf \left\{t \geq 0: u-c t-D_{t}<0\right\} .
$$

More precisely we obtain the Lundberg's estimate (19) and the value $w$ is called adjustment coefficient or Lundberg's exponent or Lundberg's parameter. Roughly speaking we show that $\psi(u)$ decays exponentially as $u \rightarrow \infty$ in the fashion of large deviations (Proposition 4.1).

By taking into account Remark 3.3 it is not restrictive to consider the Markov modulated case; indeed the classical case can be seen as a particular case.

In order to avoid the trivial case $\psi(u)=1$ for all $u>0$, the so called net profit condition is required, i.e. $c>\Lambda^{\prime}(0)$ where $\Lambda$ is defined as in (16). We point out that $\Lambda^{\prime}(0)=\sum_{i \in E} \pi_{i} \lambda_{i}\left(\mathbb{E}_{i}[X]+\right.$ $\left.\mathbb{E}_{i}[Y]\right)$.

Proposition 4.1 Assume (S2) and the net profit $c>\Lambda^{\prime}(0)$ condition hold, where $\Lambda$ is defined as in (16). Then there exists $w>0$ such that $\Lambda(w)-c w=0$ and

$$
\lim _{u \rightarrow \infty} \frac{1}{u} \log \psi(u)=-w
$$

Proof. First of all let $\Lambda_{c}$ and $\Lambda_{c}^{*}$ be the functions defined by

$$
\Lambda_{c}(\theta)=\Lambda(\theta)-c \theta \text { and } \Lambda_{c}^{*}(x)=\sup _{\theta \in \mathbb{R}}\left[\theta x-\Lambda_{c}(\theta)\right]=\Lambda^{*}(x+c)
$$

(the equality $\Lambda_{c}^{*}(x)=\Lambda^{*}(x+c)$ can be checked by the definition of $\Lambda_{c}(\theta)$ ). Then the first claim in the statement of the Proposition is the existence of $w>0$ such that $\Lambda_{c}(w)=0$.

We point out that $\Lambda_{c}^{\prime}(0)<0$ by the net profit condition $c>\Lambda^{\prime}(0)$. Moreover

$$
\Lambda_{c}(\theta) \geq \sum_{i \in E} \pi_{i} \lambda_{i}\left(\mathbb{E}_{i}\left[e^{\theta(X+Y)}\right]-1\right)-c \theta
$$

by (16), the convexity of $L$ and $\nabla L(\underline{0})=\pi$; thus $\lim _{\theta \rightarrow \infty} \Lambda_{c}(\theta)=\infty$ since the right hand side diverges as $\theta \rightarrow \infty$. In conclusion the existence of $w$ is guaranteed by the convexity of $\Lambda_{c}, \Lambda_{c}(0)=0$, $\Lambda_{c}^{\prime}(0)<0, \lim _{\theta \rightarrow \infty} \Lambda_{c}(\theta)=\infty$ and $\Lambda_{c}(\theta)<\infty$ for all $\theta \in \mathbb{R}$ (the latter statement holds by (S2) and the definition of $\left.\Lambda_{c}\right)$.

In order to prove (19) we refer to Corollary 2.3 and Lemma 2.1 in [6] (taking linear scaling functions). Then we have to check that $\Lambda_{c}^{*}$ is continuous at every point of $(0, \infty)$ and the inequality

$$
\left.\limsup _{n \rightarrow \infty} \frac{1}{n} \log \mathbb{E}\left[e^{\theta\left[\sup _{0 \leq r<1}\left(D_{n+r}-c(n+r)\right)-\left(D_{n}-c n\right)\right]}\right] \leq 0 \text { (for all } \theta>0\right) .
$$

First of all the function $\Lambda_{c}^{*}$ is convex and finite on the set $\left\{\Lambda_{c}^{\prime}(\theta): \theta \in \mathbb{R}\right\}=(-c, \infty)$; thus $\Lambda_{c}^{*}$ is continuous on this open set and in particular on $(0, \infty) \subset(-c, \infty)$. 
As far as (20) is concerned let $\theta>0$ and $n \geq 1$ be arbitrarily fixed. Then we have

$$
\begin{gathered}
\sup _{0 \leq r<1}\left(D_{n+r}-c(n+r)\right)-\left(D_{n}-c n\right)=\sup _{0 \leq r<1}\left(D_{n+r}-D_{n}-c r\right) \leq \sup _{0 \leq r<1}\left(D_{n+r}-D_{n}\right)= \\
=D_{n+1}-D_{n}=\sum_{k=1}^{N_{n+1}} X_{k}+\sum_{k \geq 1} Y_{k} 1_{T_{k}+W_{k} \leq n+1}-\left(\sum_{k=1}^{N_{n}} X_{k}+\sum_{k \geq 1} Y_{k} 1_{T_{k}+W_{k} \leq n}\right)= \\
=\sum_{k=N_{n}+1}^{N_{n+1}} X_{k}+\sum_{k \geq 1} Y_{k} 1_{n<T_{k}+W_{k} \leq n+1},
\end{gathered}
$$

whence we obtain

$$
\mathbb{E}\left[e^{\theta\left[\sup _{0 \leq r<1}\left(D_{n+r}-c(n+r)\right)-\left(D_{n}-c n\right)\right]}\right] \leq \mathbb{E}\left[\exp \left(\int_{0}^{\infty} \lambda_{J_{s}}\left(\mathbb{E}_{J_{s}}\left[e^{\theta\left[X 1_{(n, n+1]}(s)+Y 1_{(n, n+1]}(s+W)\right]}\right]-1\right) d s\right)\right]
$$

by the above inequality and by Lemma 3.2 with $\varphi(s,(x, y, w))=x 1_{(n, n+1]}(s)+y 1_{(n, n+1]}(s+w)$. The expected value in the right hand side of the latter inequality can be rewritten as

$$
\mathbb{E}\left[\exp \left(\int_{0}^{n} \lambda_{J_{s}}\left(\mathbb{E}_{J_{s}}\left[e^{\theta Y 1_{(n-s, n+1-s]}(W)}\right]-1\right) d s+\int_{n}^{n+1} \lambda_{J_{s}}\left(\mathbb{E}_{J_{s}}\left[e^{\theta\left[X+Y 1_{(n-s, n+1-s]}(W)\right]}\right]-1\right) d s\right)\right]
$$

and, by considering the change of variable $u=n-s$ in both the integrals, the latter is equal to

$$
=\mathbb{E}\left[\exp \left(\int_{0}^{n} \lambda_{J_{n-u}}\left(\mathbb{E}_{J_{n-u}}\left[e^{\theta Y 1_{(u, u+1]}(W)}\right]-1\right) d u+\int_{-1}^{0} \lambda_{J_{n-u}}\left(\mathbb{E}_{J_{n-u}}\left[e^{\theta\left[X+Y 1_{(u, u+1]}(W)\right]}\right]-1\right) d u\right)\right] .
$$

Now set $\left.M(\theta)=\max _{i \in E} \lambda_{i}\left(\mathbb{E}_{i}\left[e^{\theta(X+Y)}\right]-1\right)\right)$ and $M(\theta)<\infty$ by (S2); then we have

$$
\mathbb{E}\left[e^{\theta\left[\sup _{0 \leq r<1}\left(D_{n+r}-c(n+r)\right)-\left(D_{n}-c n\right)\right]}\right] \leq \exp \left(\int_{0}^{n} \max _{i \in E}\left\{\lambda_{i}\left(\mathbb{E}_{i}\left[e^{\theta Y 1_{(u, u+1]}(W)}\right]-1\right)\right\} d u+M(\theta)\right),
$$

whence we obtain

$$
\begin{gathered}
\limsup _{n \rightarrow \infty} \frac{1}{n} \log \mathbb{E}\left[e^{\theta\left[\sup _{0 \leq r<1}\left(D_{n+r}-c(n+r)\right)-\left(D_{n}-c n\right)\right]}\right] \leq \\
\leq \limsup _{n \rightarrow \infty} \frac{1}{n}\left(\int_{0}^{n} \max _{i \in E}\left\{\lambda_{i}\left(\mathbb{E}_{i}\left[e^{\theta Y 1_{(u, u+1]}(W)}\right]-1\right)\right\} d u+M(\theta)\right)= \\
=\limsup _{n \rightarrow \infty} \frac{1}{n} \int_{0}^{n} \max _{i \in E}\left\{\lambda_{i}\left(\mathbb{E}_{i}\left[e^{\theta Y 1_{(u, u+1]}(W)}\right]-1\right)\right\} d u .
\end{gathered}
$$

In conclusion (20) holds since $\lim _{n \rightarrow \infty} \frac{1}{n} \int_{0}^{n} \max _{i \in E}\left\{\lambda_{i}\left(\mathbb{E}_{i}\left[e^{\theta Y 1_{(u, u+1]}(W)}\right]-1\right)\right\} d u=0$ by Hopital rule.

\section{Acknowledgements}

This work has been partially supported by Murst Project "Metodi Stocastici in Finanza Matematica". 


\section{References}

[1] Baldi P. and Piccioni M., 1999, A representation formula for the large deviation rate function for the empirical law of a continuous time Markov chain. Statist. Probab. Lett., 41, 107-115.

[2] Borovkov A.A., 1967, Boundary values problems for random walks and large deviations for function spaces. Theory Probab. Appl., 12, 575-595.

[3] Brémaud P., 2000, An insensitivity property of Lundberg's estimate for delayed claims. J. Appl. Prob., 37, 914-917.

[4] de Acosta A., 1995, Large deviations for vector valued Lévy processes. Stochastic Process. Appl., 51, 75-115.

[5] Dembo A. and Zeitouni O., 1993, Large Deviations Techniques and Applications (Boston: Jones and Bartlett).

[6] Duffield N.G. and O'Connell N., 1995, Large deviations and overflow probabilities for a single server queue, with applications. Math. Proc. Camb. Phil. Soc., 118, 363-374.

[7] Ganesh A., Macci C. and Torrisi G.L., 2005, Sample path large deviations principles for Poisson shot noise processes, and applications. Electron. J. Probab., 10, 1026-1043.

[8] Macci C., Stabile G. and Torrisi G.L., 2005, Lundberg parameters for non standard risk processes. Scand. Actuar. J., 2005, 417-432.

[9] Macci C. and Torrisi G.L., 2004, Asymptotic results for perturbed risk processes with delayed claims. Insurance Math. Econom., 34, 307-320.

[10] Rolski T., Schmidli H., Schmidt V. and Teugels J.L., 1999, Stochastic Processes for Insurance and Finance (Chichester, Wiley).

[11] Yuen K.C., Guo J. and Ng K.W., 2005, On ultimate ruin in a delayed-claims model. J. Appl. Prob., 42, 163-174. 